(1985) Pferdeheilkunde 1, 113-118

\section{Endokrinologie von Fötus und Stute während der späten Trächtigkeitsphase und Geburt}

\author{
R. L. Pashen
}

Department of Reproduction, School of Veterinary Medicine, University of California

\section{Einleitung}

Das Ende der Trächtigkeit, die Geburt und die plötzlich auftretende Notwendigkeit, sich an die extrauterine Umgebung anzupassen, machen die perinatale Periode bei allen Spezies zu einer ereignisreichen Phase. Die vielen funktionellen und entwicklungsbedingten Veränderungen, die zu dieser Zeit ablaufen, sind wahrscheinlich bedeutender als in jedem anderen Lebensabschnitt. In dieser Arbeit werden die endokrinologischen Veränderungen im späten Teil der Gravidität der Stute in ihrer Beziehung zum Beginn der Geburt untersucht. Leider weiß man wenig Vergleichbares über hormonale Entwicklungen beim Fötus vor der Geburt. Bevor auf die spezifischen Hormonmuster der präpartalen Phase eingegangen wird, ist es wichtig, einen allgemeinen Überblick über die endokrinologischen Hauptvorgänge der Trächtigkeit beim Pferd vorauszuschicken; er ist in Abb. 1 dargestellt.

\section{Gestagene}

Die Veränderungen im Plasma-Progesteronspiegel während der ersten Graviditätshälfte spiegeln direkt die materne Ovarfunktion wider. Anfangs sekretiert das primäre Corpus luteum Progesteron, aber nach dem 35. Tag steuern akzessorische Corpora lutea, die sich unter dem gemeinsamen Einfluß von FSH und equinem Chorion-Gonadotropin gebildet haben, in zunehmendem Maß zum Pool des zirkulierenden Progesterons bei (Cole et al., 1931; Amoroso et al., 1948; Squires et al., 1975; Urwin et al., 1982). Auch die Plazenta bildet etwa vom 50. Tag an Progesteron; sie ist in der Zeit vom 150. bis 200. Trächtigkeitstag, wenn die Corpora lutea ihre Funktion eingestellt haben, die einzige Progesteronquelle zur Aufrechterhaltung der Gravidität (Holtan et al., 1979). Barnes et al. (1975) zeigten an Föten mit Dauerkatheter, daß die Progesteronspiegel des fötalen Blutkreislaufs viel höher als die des maternen Plasmas sind. Während der letzten 100 Trächtigkeitstage lagen die in der Umbilikalvene gemessenen Konzentrationen um 15 bis $20 \mathrm{ng} / \mathrm{ml}$ höher als die im Blut aus der Umbilikalarterie, wogegen die Progesteronspiegel im Blut der Uterusvenen gleichbleibend höher als die des maternen peripheren Blutkreislaufs waren. Beim fötalen Plasma-Progesteron wa- ren mit herannahender Geburt keine deutlichen Veränderungen $\mathrm{zu}$ beobachten.

Im Gegensatz zum Fötus bleiben die maternen peripheren Plasma-Progesteronspiegel um die Mitte der Gravidität bis zu den letzten 30 bis 60 Trächtigkeitstagen niedrig. Dann beginnt die Konzentration stetig zu steigen, um nach der Geburt des Fohlens auf die Ausgangshöhe abzusinken (Abb. 2). Mit dem Anwachsen der Progesteronwerte ist eine Erhöhung der Blutspiegel einer Anzahl eng verwandter Gestagene verbunden (Holtan et al., 1975; Burns et al., 1975; Seamans et al., 1979). Man hat diese in neuerer Zeit identifiziert, und es gehören die $5 \alpha$-Pregnan-Metaboliten des Progesterons dazu, $5 \alpha$-Pregnan-3, 20-dion (DHP), $3 \beta$ Hydroxy-5 $\alpha$-Pregnan-20-on ( $3 \beta$-ol), $20 \alpha$-Hydroxy-5 $\alpha$-Pregnan-3-on (20 $\alpha$-ol) (Seamans et al., 1979) sowie 20 $\alpha$-Dihydroprogesteron und $17 \alpha$-Hydroxyprogesteron (Barnes et al., 1975; Holtan et al., 1975). Moss et al. (1979) untersuchten die Herkunft dieser Verbindungen und fanden, daß DHP hauptsächlich in der Plazenta und einem maternen Syntheseort gebildet wird, daß $20 \alpha$-ol ebenfalls in erster Linie maternen Ursprungs ist und daß $3 \beta$-ol fast ausschließlich vom Fötus produziert wird. Barnes et al. (1975) erstellten die These, daß 20 $\alpha$-Dihydroprogesteron auch fast in seiner gesamten Menge im Fötus ensteht. Die biologische Funktion dieser Metaboliten ist unbekannt, aber ihre schrittweise Konzentrationserhöhung im Blutkreislauf in der späten Trächtigkeitsphase läßt annehmen, daß kurz vor der Geburt generell eine Änderung im Progesteronstoffwechsel abläuft. Ihre Bedeutung wird später besprochen.

\section{Östrogene}

In der zweiten Graviditätshälfte bestehen hohe Östrogenkonzentrationen im Blut und Urin von Stuten. Ihr Kurvenverlauf weist Spitzen im 7. und 8. Monat der Trächtigkeit auf, um zum Geburtstermin hin schrittweise abzufallen. Beim Fötus sind die Plasma-Östrogenkonzentrationen höher als bei der Stute, und der Spiegel des Umbilikalvenenbluts liegt über dem des arteriellen Plasmas des Fötus (Barnes et al., 1975).

Bei der Gravidität des Pferdes trifft man auf zwei Hauptgruppen von Östrogenen; es sind die weitverbreiteten phenolischen Östrogene Östron und Östradiol-17 $\beta$ sowie die am Ring B ungesättigten Östrogene Equilin und Equilenin, die eine Besonderheit beim Pferd darstellen. Die Konzentration im Blutkreislauf jedes dieser Östrogene ändert sich unabhängig von der der anderen, und es gibt, wie Abb. 3 zeigt, Hinweise dafür, daß ihre Produktion über unterschiedliche Synthesewege ablaufen kann (Starka, 1966; Bhavnani et al., 1971; Short et al., 1971; Bhavnani et al., 1978). Die durchlaufenen exakten Reaktionswege sind weiterhin Gegenstand von Überlegungen, fest steht aber, daß die fötalen Gonaden, die parallel zum Anstieg der Östrogenwerte dramatisch an Größe zunehmen, Vorläufer liefern, die schließlich in der Plazenta in Östrogene umgewandelt werden (Pashen et al., 1979; Pashen et al., 1982). Die Gonaden produzieren Dihydroepiandrosteron (DHA), das von der Plazenta zu Östron und Östradiol-17 $\beta$ metabolisiert wird. Ob DHA auch der direkte Vorläufer von Equi- 

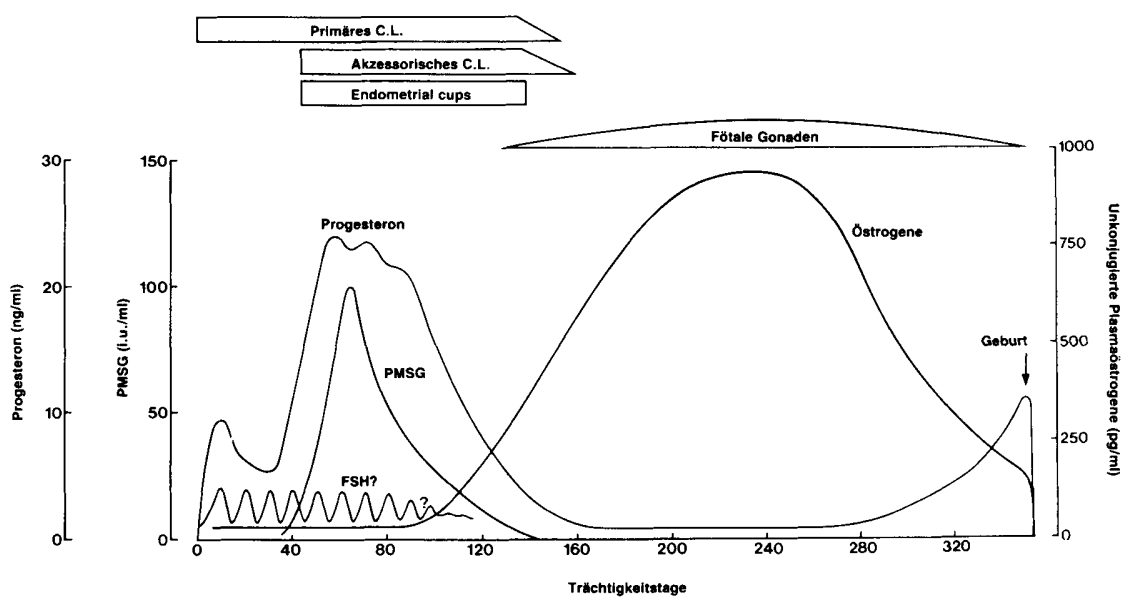

Abb. 1: Graphische Darstellung der Kontrollfaktoren von Gonadotropin- (Pregnant-Mare's Serum-Gonadotropin und Follikelstimulierendes Hormon) und Steroid- (Progesteron und Östrogen)Konzentrationen im maternen peripheren Plasma während der Trächtigkeit bei der Stute. lin ist, ist unklar. Möglicherweise wird es erst in der fötalen Leber transformiert, bevor es die Plazenta zu Equilin umwandelt (Starka et al., 1966; Pashen et al., 1982). Andererseits könnten die Gonaden eine DHA-ähnliche Substanz sekretieren, die die Plazenta direkt zu Equilin metabolisiert (Abb. 3) (Pashen et al., 1982; Tait et al., 1983). Jedenfalls existiert eine echte fötoplazentare Produktionseinheit für Östrogen beim Pferd, und zur Östrogen-Biosynthese bedarf es sowohl des Föten als auch der Plazenta. Beim Menschen besteht eine ähnliche Beziehung zwischen der fötalen Nebenniere und der Plazenta, und klinisch macht man von der Verbindung vorteilhafterweise Gebrauch, indem man am Monitor die Gesundheit und Lebensfähigkeit der fötoplazentaren Einheit als Ganzer überwacht. Rance et al. (1978) schlugen vor, den Östrogenspiegel beim Pferd diagnostisch zu nutzen, um zu beurteilen, ob die Gravidität physiologisch verläuft. Angaben konnten dazu aber wegen der großen individuellen Schwankungsbreite der Werte bei normalen Stuten und der unterschiedlichen Bestimmungsmethoden, welche die verschiedenen Labors zur Östrogenmessung heranziehen, nicht gemacht werden.

Nichtsdestoweniger wiesen jedoch die Experimente, die die Rolle der Gonaden bei der Östrogenproduktion in ihrer Bedeutung herausstellten, auch auf die Funktion hin, welche u. U. die sowohl im fötalen als auch im maternen Kreislauf während der Trächtigkeit bestehenden hohen
Östrogenspiegel haben. Im Mittelpunkt der Untersuchungen stand die chirurgische Entfernung der fötalen Gonaden im mittleren bis späten Abschnitt der Gravidität, deren Ergebnis ein plötzlicher, überstürzter Abfall der Östrogenspiegel auf Werte nahe den Ausgangskonzentrationen war (Pashen et al., 1979). Die Trächtigkeiten bestanden trotz der niedrigen Östrogenspiegel etwa in physiologischer Dauer fort (Abb. 4). Nach einer normalen Geburtseinleitung waren dann aber die Wehen bei den Stuten, die gonadektomierte Föten trugen, schwach und ohne Wirkung, und auch die Prostaglandinspiegel waren niedriger als gewöhnlich. Östrogene scheinen also während der Wehen an der Prostaglandinproduktion beteiligt zu sein. Ein solcher Befund stimmt mit den engen Verbindungen überein, die, wie man weiß, zwischen einer Zunahme der Östrogenkonzentration und der Stimulierung der Prostaglandinsynthese beim Schaf um den Geburtszeitpunkt herum bestehen (Thorburn et al., 1972; Mitchell et al., 1979).

Weiterhin fand man bei der Untersuchung heraus, daß gonadektomierte Fohlen von Stuten mit zu niedrigen Östrogenspiegeln bei der Geburt unreif waren. Hull et al. (1976) zeigten, daß eine ähnliche Beziehung beim Menschen existiert, indem sie bei schwangeren Frauen einen Zusammenhang zwischen „für den Geburtszeitpunkt kleinen“ oder unreifen Neugeborenen und unnormal niedrigen Östrogenspiegeln nachwiesen. Es ist nicht geklärt, wie diese Ef-

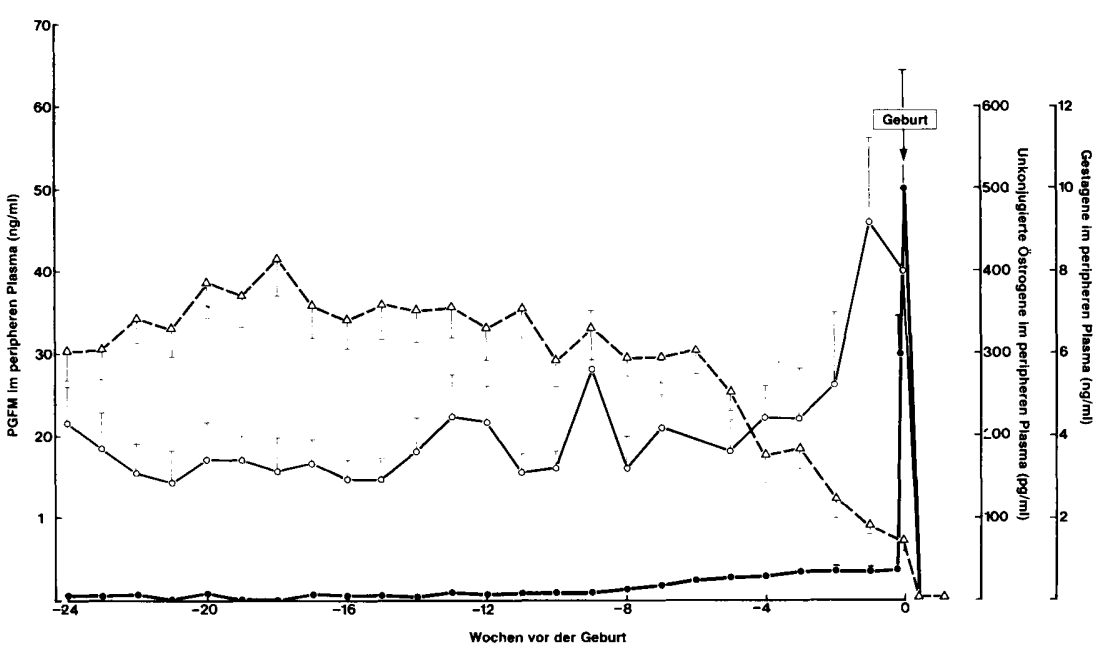

Abb. 2: Mittlere ( \pm Standardabweichung vom Mittelwert) Plasma-Konzentrationen von Progesteron $(\mathrm{O})$, unkonjugiertem Östrogen $(\Delta)$ und PGFM (O) bei acht Ponystuten während der zweiten Graviditätshälfte und der Spontangeburt (Pashen, 1981) 


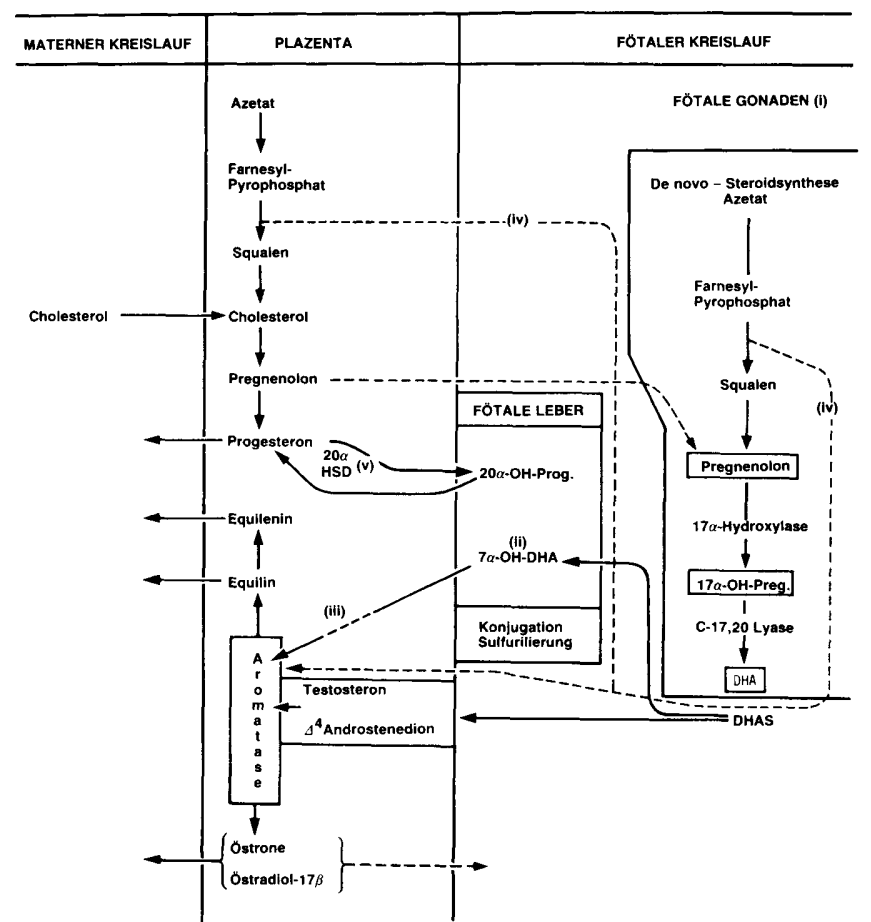

Abb. 3: (i) Fötale Gonaden: zur De-novo-Steroid-Synthese aus Azetat befähigt; Mangel an $\Delta^{5} 3 \beta \mathrm{HSD}$ verhindert Umwandlung von Pregnenolon in Progesteron; hoher venös-arterieller DHA-Gradient über die Gonaden hinweg läßt DHA-Sekretion durch sie vermuten. (ii) Hypothetische $7 \alpha$-Hydroxylierung von DHA in der fötalen Leber mit $7 \alpha-\mathrm{OH}$ DHA, das möglicherweise als obligater Vorläufer von Equilin funktioniert. (iii) Reihe der plazentaren Enzyme, die wahrscheinlich an der Umwandlung von $7 \alpha-\mathrm{OH}-\mathrm{DHA}$ in die am Ring B ungesättigten Östrogene beteiligt sind. (iv) Unkonventioneller Steroid-Biosyntheseweg, wie von B. R. Bhavnani und Mitarbeitern für die Steroid-Produktion vorgeschlagen. (v) Recycling von $20 \alpha$-Dihydroprogesteron zwischen Fötus und Plazenta als Progesteron-Quelle. Erklärung der Abkürzungen: DHA Dihydroepiandrosteron; DHAS Dihydroepiandrosteronsulfat; $7 \alpha$ OH-DHA $7 \alpha$-Hydroxy-DHA; 20 $\alpha$-HSD $20 \alpha$-Hydroxysteroid-Dihydrogenase; $20 \alpha-\mathrm{OH}$-Prog $20 \alpha$-Dihydroprogesteron; $17 \alpha$-OH-Preg $17 \alpha$ Hydroxypregnenolon. fekte gesteuert werden, aber beim Schaf können Östrogene und ihre Vorläufer Grad und Verteilung des Blutflusses in Uterus und Plazenta regulieren (Resnik et al., 1974; Nuway. bid et al., 1975; Pupkin et al., 1975; Rosenfeld et al., 1978). Niedrige Östrogenkonzentrationen können daher auf den uterinen und plazentaren Blutfluß in einer Weise hemmend einwirken, daß die transplazentare Übertragung essentieller Grundstoffe vermindert und Permeabilität und Stoffwechsel der Plazenta verändert sind.

\section{Relaxin}

Man hat in neuerer Zeit demonstriert, daß etwa vom 80 . Trächtigkeitstag bis zur Geburt das Serum von Stuten Relaxin in steigender Konzentration enthält (Stewart et al., 1981; Stewart et al., 1982). Das Synthesemuster des Relaxins folgt eng dem der Östrogene, obwohl keine Verbindung zwischen den beiden festgestellt werden konnte. Wahrscheinlich spielt Relaxin eine synergistische Rolle mit Progesteron bei der Ruhigstellung des Uterus während der Gravidität.

\section{Prolaktin}

Die Prolaktinspiegel sind bei der Stute in der Trächtigkeit sehr unterschiedlich und weisen keinen klaren Produktionsplan im Zusammenhang mit dem Einsetzen der Laktation oder der Geburt auf (Forsyth et al., 1975; Nett et al., 1975).

\section{Plazentares Laktogen}

Bisher konnte man bei der trächtigen Stute Laktogen nicht nachweisen (Forsyth et al., 1975).

\section{Geburt}

Bekanntlich bestimmt bei vielen Spezies der Fötus den Geburtszeitpunkt, und diese Kontrolle wird auf dem Weg ei-

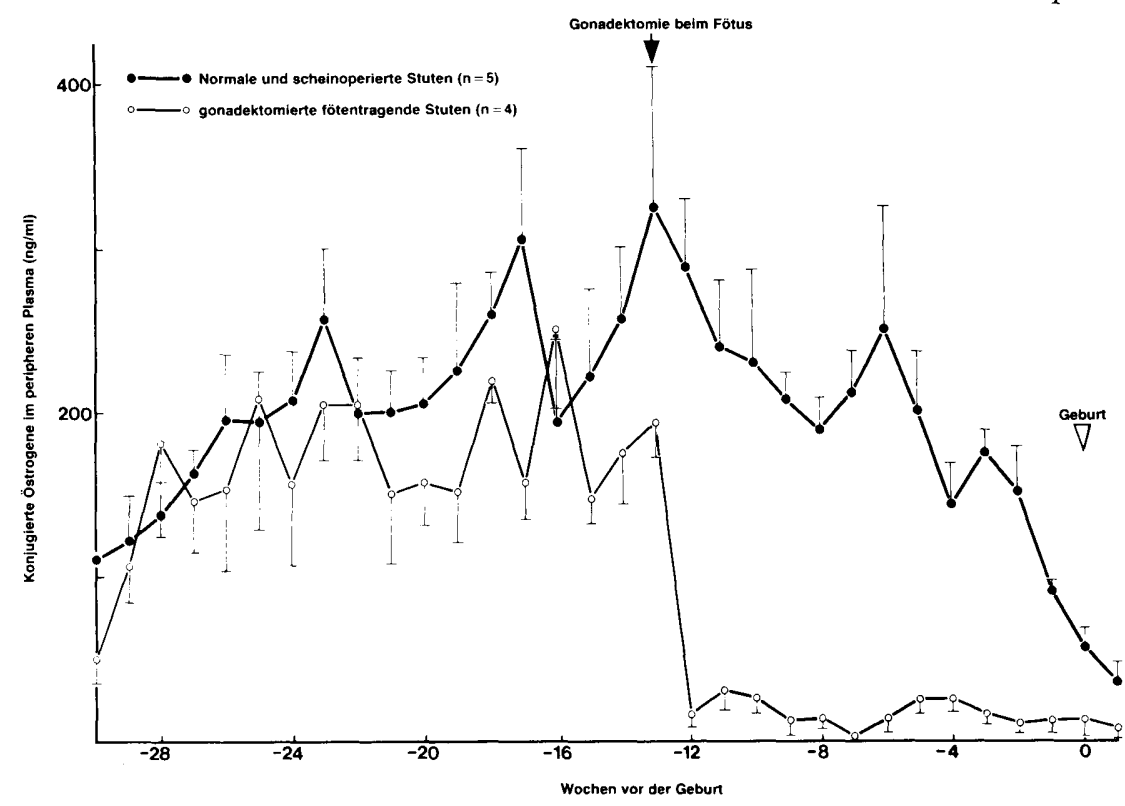

Abb. 4: Mittlere ( \pm Standardabweichung vom Mittelwert) Konzentrationen von konjugiertem Östrogen im peripheren Plasma von Stuten mit normalen und scheinoperierten (0) und gonadektomierten (O) Föten in der zweiten Graviditätshälfte (Pashen et al., 1979). 


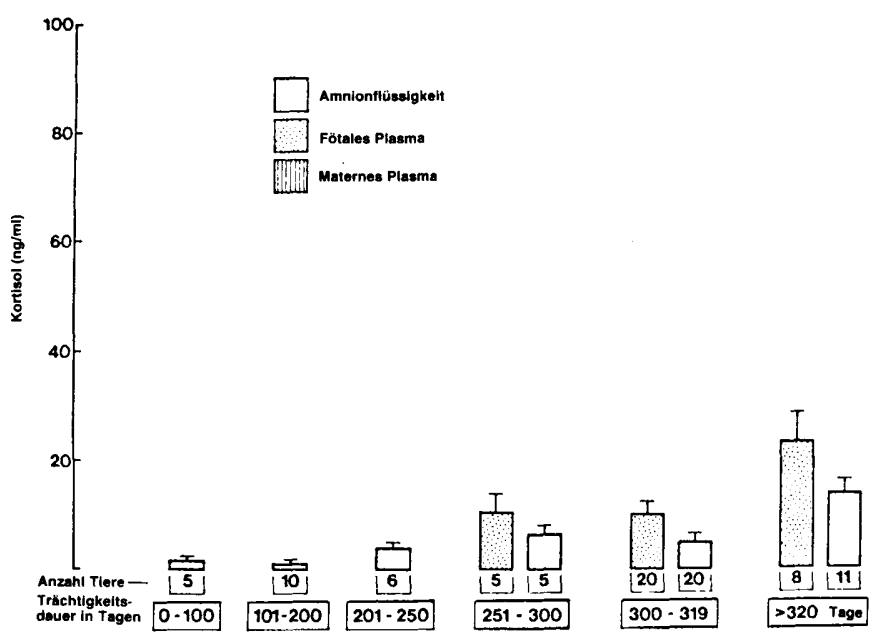

ner Zunahme der fötalen Kortisolsynthese gesteuert, die einen direkten Effekt der Reifung der fötalen Hypothalamus-Hypophysenvorderlappen-Nebennieren-Achse darstellt. Die Ausreifung dieses Systems hat sich als wesentlich bei der Regulierung der Lungenentwicklung, der Freisetzung des Surfactant-Faktors und der Aktivierung von Enzymsystemen im Verdauungstrakt, in der Leber und der Plazenta erwiesen (Moog, 1970); Liggins et al., 1981).

Diese Veränderungen sind am besten beim Schaf belegt, wo Kortisol etwa drei Tage vor der Geburt plötzlich in einem Schwall ansteigt (Bassett et al., 1969; Comline et al., 1970; Thorburn et al., 1979). Das Anwachsen des fötalen Kortisolspiegels führt zur Aktivierung von plazentaren Enzymsystemen, die eine direkte Umwandlung von Progesteron in Östrogene erlauben (Flint et al., 1979) und im Endeffekt einen Abfall der Progesteronkonzentration und einen rapiden Anstieg der Östrogenwerte bewirken. Dies wiederum regt an, daß die Prostaglandin $\mathrm{F}_{2 \alpha}$-Produktion zunimmt und die Wehen einsetzen (Challis et al., 1972; Flint et al., 1974). Beim Pferd hat man solche dramatischen Veränderungen wegen der Schwierigkeiten im Zusammenhang mit Legen und Belassen von Kathetern bei fötalen Fohlen kurz vor der Geburt nicht nachgewiesen (Silver, 1980). So weiß man nicht, ob der Pferdefötus ebenfalls über die Reifung der fötalen Hypophysenvorderlappen-Nebennieren-Achse die Kontrolle über seine Geburt ausübt. Die Kortisolspiegel im maternen und fötalen Blut und in den Körperflüssigkeiten des Fötus beginnen mit herannahendem Geburtstermin zu steigen (Abb. 5) (Pashen, 1981), und es liegen Berichte über angehobene fötale Plasma-Kortisolkonzentrationen ganz kurz vor der Geburt vor (Nathanielsz et al., 1975). Eine spezifische Anhebung der Kortisolwerte wurde jedoch bisher nicht mit der Geburt direkt in Zusammenhang gebracht, wenn auch wahrscheinlich kurz vor der Entbindung allgemein eine Erhöhung des fötalen Kortisolspiegels eintritt. Mit Sicherheit beantwortet aber die Nebenniere bei Fohlen, die nach Ablauf der physiologischen Trächtigkeitsdauer geboren wurden, bei Untersuchungen in der Zeit nach der Geburt exogenes adrenokortikotropes Hormon stärker als bei geburtsunreifen Fohlen (Pashen, 1981); Rossdale et al., 1982). Beim termingerecht geborenen Foh-

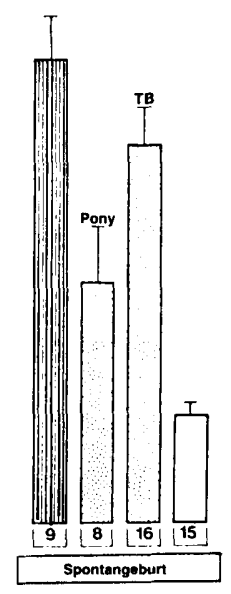

Abb. 5: Mittlere ( \pm Standardabweichung vom Mittelwert) Kortisolkonzentrationen in Proben von Amnionflüssigkeit und fötalem und maternem Plasma, in verschiedenen Stadien der Trächtigkeit entnommen. Das Material besteht aus Praxisfällen, aus graviden Stuten und Föten vom Schlachthof oder aus laufenden Experimenten. Die Probennahme nach dem 320. Trächtigkeitstag wurde an Stuten durchgeführt, deren Geburt mit Oxytozin oder Fluprostenol eingeleitet wurde (Pashen, 1981).

len steigt der endogene Kortisolspiegel direkt nach der Geburt, ein Hinweis darauf, daß die Nebenniere in der Lage ist, auf den Streß der postnatalen Adaptationsphase zu reagieren. Es gibt eine ganze Anzahl von Möglichkeiten, wie fötales Kortisol beim Pferd als Hauptkontrollfaktor der Geburt agieren könnte. Vielleicht ist der veränderte Progesteron-Metabolismus der späten Graviditätsphase das Ergebnis einer kortisol-induzierten Abwandlung plazentarer Enzyme, die zur Synthese biologisch "schwacher" Gestagene führt. Diese Gestagene könnten u. U. die Rezeptorbindungsstellen im Myometrium besetzen und dadurch effektiv einen Progesteronentzug bewirken. Die Folge wäre ein Anstieg der Aktivität und Sensibilität des Myometriums. Die Geburt läuft bei der Stute verglichen mit den anderen großen Haustierarten extrem rasch ab und dauert gewöhnlich etwa 45 Minuten (Rossdale et al., 1974).

Für viele Veränderungen im Organismus gibt es deutliche Belege, die darauf hinweisen, daß das Fohlen bald zur Welt kommt, und das Kolostrum im Euter, die Harztröpfchen an den Zitzen, die verminderte Viskosität des Zervikalschleims und die Erschlaffung der Zervix, all dies zeigt an, daß wichtige hormonelle Vorgänge ablaufen, die die Stute auf die Geburt vorbereiten.

Wie bereits besprochen, ändern sich die maternen peripheren Hormonkonzentrationen in der Zeit unmittelbar vor der Geburt nicht wesentlich. Vielmehr neigt der Progesteronspiegel ganz kurz vor dem Einsetzen der Wehen zu einer stetigen $\mathrm{Zu}$ - und der Östrogenspiegel zu einer Abnahme (Abb. 2). Dieser Zustand ähnelt dem beim Menschen und anderen Primaten, die ebenfalls ohne größere Veränderungen der maternen peripheren Hormonwerte gebären. Für diese Spezies hat man die These aufgestellt, daß Abwandlungen in Typ und Anzahl von Rezeptoren, Verfügbarkeit von Bindungsproteinen und andersartigem Stoffwechsel der Hormone hauptsächlich zu geänderten Hormonkonzentrationen auf der Ebene der Gewebe führen (Thorburn et al., 1979). Nur die Untersuchungen von Barnes et al. $(1975,1978)$ deuten darauf hin, daß u. U. ähnliche Veränderungen beim Pferd ablaufen. Diese Autoren zeigten, daß die Progesteronkonzentration im Umbilikalkreislauf 24 bis 48 Stunden vor der Entbindung signifikant ab- 

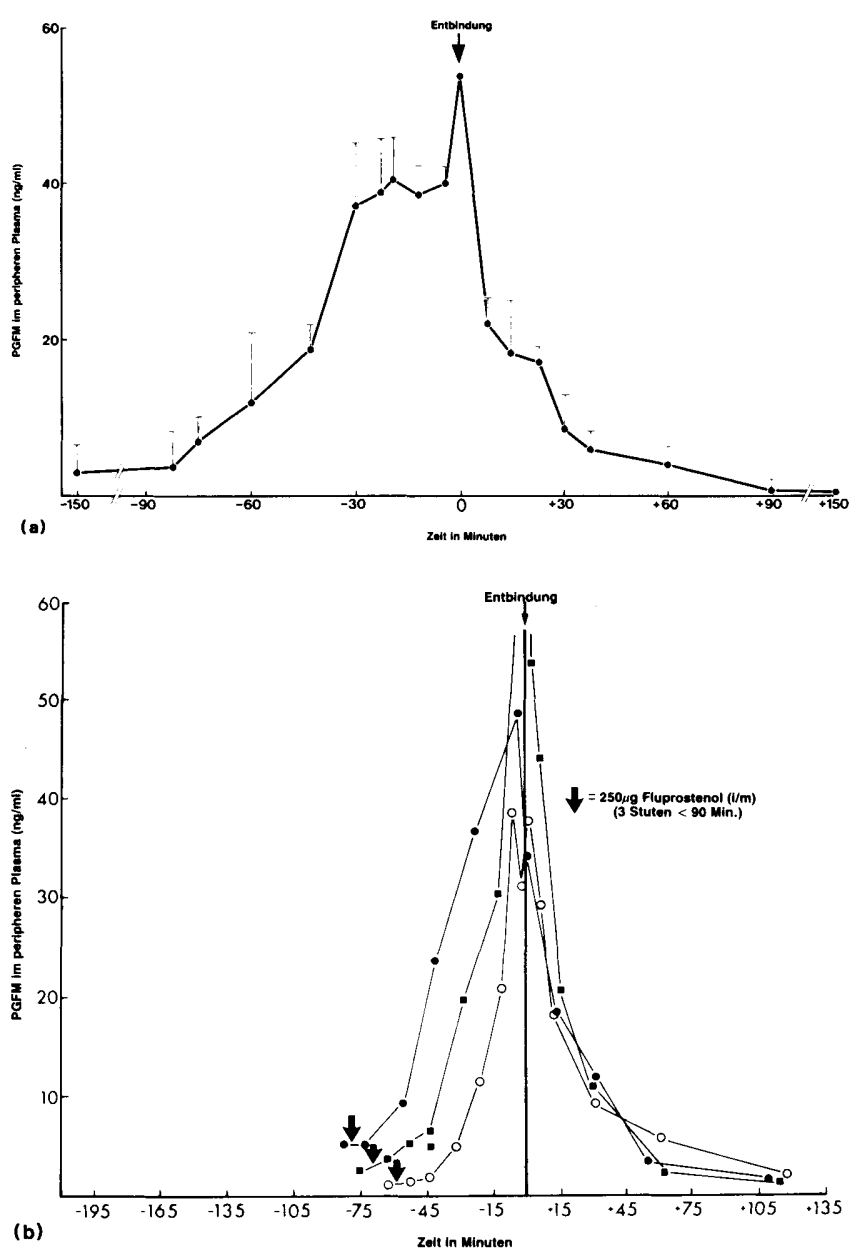

Abb. 6: (a) Mittlere ( \pm Standardabweichung vom Mittelwert) periphere Plasma-PGFM-Konzentrationen bei acht Stuten während ihrer Spontangeburt. Beachte den anfänglichen Anstieg parallel zum Einsetzen des ersten Wehenstadiums und die zweite rasche Zunahme im Zusammenhang mit dem zweiten Wehenstadium (Pashen et al., 1979). (b) Plasma-PGFM-Spiegel bei Stuten nach Geburtseinleitung mit 250 $\mu \mathrm{g}$ des synthetischen Prostaglandin-Analogs Fluprostenol. Die vertikale Linie bezeichnet den Zeitpunkt, an dem die Geburt abgeschlossen war (Pashen, 1981).

fällt, obwohl nur geringe Abweichungen des maternen Plasma-Progesteronspiegels der Geburt vorausgehen. Weiterhin wächst die materne Östradiol-17 $\beta$-Konzentration im Gegensatz zu den gleichzeitig absinkenden Spiegeln von Östron und Equilin zum Geburtszeitpunkt an. Die Bedeu-

\section{Literatur}

Allen, W. E., Chard, T., und Forsling, M. L. (1973): Peripheral plasma levels of oxytocin and vasopressin in the mare during parturition. J. Endocrin. 57, 175-176.

Amoroso, E. C., Hancock, J. L., und Rowelands, I. W. (1948): Ovarian activity in the pregnant mare. Nature (Lond.). 161, 355-356.

Barnes, R. J., Comline, R. S., Jeffcott, L. B., Mitchell, M. D., Rossdale, P. D., und Silver, $M$. (1978): Foetal and maternal plasma concentrations of 13, 14-Dihydro-15-oxo-prostaglandin $F$ in the mare during late pregnancy and at parturition. J. Endocrin. 78, 201-215.

Barnes, R. J., Nathanielsz, P. W., Rossdale, P. D., Comline, R. S., und Silver, $M$. (1975): Plasma progestagens and oestrogens in fetus and mother in late pregnancy. J. Reprod. Fert. Supple. 23, 617-623. tung dieses späten Anstiegs der Östradiol-17 $\beta$-Werte ist zwar unklar, er kann aber ein wichtiger Anreiz für die Prostaglandin $\mathrm{F}_{2 \alpha}$-Synthese und -Freisetzung sein, wenn das Fohlen zur Welt kommt. Studien von Currie et al. (1973) und Flint et al. (1974) ließen erkennen, daß Östradiol-17 $\beta$ beim Schaf zweifellos ein wirkungsvolles Östrogen bei der Induzierung der Prostaglandin $\mathrm{F}_{2 \alpha}$-Synthese in der präpartalen Phase ist.

Prostaglandine sind beim Wehenverlauf der Stute auch von Bedeutung. Der Spiegel von Prostaglandin $\mathrm{F}_{2 \alpha}$ (Sharma, 1975; Silver et al., 1979) und seinem zirkulierenden Hauptmetaboliten, 13, 14-Dihydro-15-oxo-PGF ${ }_{2 \alpha}$ (PGFM) (Barnes et al., 1978; Silver et al., 1979; Pashen et al., 1979) wächst im maternen Blutkreislauf in den letzten Graviditätswochen und während der Wehen an, und mit dem synthetischen Prostaglandin-F-Analog Fluprostenol kann zum physiologischen Zeitpunkt die Geburt eingeleitet werden (Rossdale et al., 1979).

Im ersten Wehenstadium beginnen die Plasma-PGFM-Konzentrationen zuzunehmen und zeigen damit eine gesteigerte Prostaglandin- $\mathrm{F}_{2 \alpha}$-Produktion an, die die Aktivität des Myometriums erhöht und den Uterus für die weitere Prostaglandinfreisetzung sensibilisiert. Diese Phase dauert 20 Minuten bis 3 Stunden, gefolgt von einem explosiven Anstieg der Prostaglandinproduktion und dem synchronen Einsetzen des zweiten Wehenstadiums (Abb. 6) (Pashen et al., 1979).

Die Prostaglandine spielen vermutlich bei der Wehenmechanik der Stute eine fundamentale Rolle; Versuche, die Trächtigkeit durch Hemmung der Prostaglandinsynthese $\mathrm{zu}$ verlängern, waren bisher jedoch nicht erfolgreich $(\mathrm{Pa}$ shen et al., 1981). Die Plasma-Oxytozin-Titer wachsen im zweiten Wehenstadium ebenfalls an (Allen et al., 1973), wahrscheinlich als Effekt einer Dehnung von Zervix und Vagina beim Eintritt des Fötus in den Geburtskanal. Außerdem treibt, wie man weiß, exogenes Oxytozin die PGFM-Konzentration rasch in die Höhe (Barnes et al., 1978) und initiiert den Geburtsvorgang (Hillman, 1975; Purvis, 1978; Barnes et al., 1978; Pashen, 1981). Daher darf man wohl annehmen, daß wie beim Schaf Oxytozin und Prostaglandin F synergistisch den Anreiz für die kräftigen rhythmischen Uteruskontraktionen des zweiten Wehenstadiums geben, die gewährleisten, daß das Fohlen geboren wird.

Bassett, J. M., und Thorburn, G. D. (1969): Foetal plasma corticosteroids and the initiation of parturition in sheep. J. Endocrin. 44, 285-286.

Bhavnani, B. R., Short, R. V., und Solomon, S. (1971): Formation of estrogens by the pregnant mare: II Metabolism of ${ }^{14} \mathrm{C}$ acetate and ${ }^{3} \mathrm{H}$ cholesterol injected into the fetal circulation. Endocrin. 89, 1152-1157.

Bhavnani, B. R., und Woolever, C. A. (1978): Formation of steroids by the pregnant mare: VI Metabolism of $\left({ }^{14} \mathrm{C}\right)$ farnesyl pyrophosphate and $\left({ }^{3} \mathrm{H}\right)$ dehydroepiandrosterone injected into the fetus. Endocrin. 103, 2291-2298.

Burns, S. J., und Fleeger, J. L. (1975): Plasma progestagens in the pregnant mare in the first and last 90 days of gestation. J. Reprod. Fert. Supple. $23,435-439$.

Challis, J. R. G., Harrison, F. A., Heap, R. B., Horton, E. W., und Poyser, N. $L$. (1972): A possible role of oestrogens in the stimulation of $\mathrm{PGF}_{2 \alpha}$ output at the time of parturition in a sheep. J. Reprod. Fert. 30, 485-488. 
Cole, H. H., Howell, C. E., und Hart, G. H. (1931): The changes occurring in the ovary of the mare during pregnancy. Anat. Rec. 49, 199-209.

Comline, R. S., Nathanielsz, P. W., Paisey, R. B., und Silver, M. (1970): Cortisol turnover in the sheep foetus immediately prior to parturition. J. Physiol. 210, 141-142.

Currie, W. B., Wong, M. S. F., Cox, R. F., und Thorburn, G. D. (1973): Spontaneous or dexamethasone induced parturition in the sheep and goat; changes in plasma concentrations of maternal prostaglandin $\mathrm{F}$ and foetal oestrogen sulphate. Mem. Soc. Endocr. 20, 95-118.

Flint, A. P. F., Anderson, A. B. M., Patten, P. T., und Turnbull, A. C. (1974): Control of utero-ovarian venous prostaglandin $\mathrm{F}$ during labour in sheep - acute effects of vaginal and cervical stimulation. J. Endocrin. 63, 67-87.

Flint, A. P. F., Ricketts, A. P., und Craig, V. A. (1979): The control of placental steroid synthesis at parturition in domestic animals. An. Reprod. Sci. 2, 239-251.

Forsyth, J. A., Rossdale, P. D., und Thomas, C. R. (1975): Studies on milk composition and lactogenic hormones in the mare. J. Reprod. Fert. Supple. 23, 631-635.

Hay, M. F., und Allen, W. R. (1975): An ultrastructural and histochemical study of the interstitial cells in the gonads of the fetal hores. J. Reprod. Fert. Supple. 23, 557-561.

Hillman, $R$. B. (1975): Induction of parturition in mares. J. Reprod. Fert. Supple. 23, 641-644.

Holtan, D. W., Nett, T. M., und Estergreen, V. L. (1975): Plasma progestins in pregnant, post partum and cycling mares. J. Anim. Sci. 40, 251-260.

Holtan, D. W., Squires, E. L., Lapin, D. R., und Ginther, O. J. (1979): Effect of ovariectomy on pregnancy in mares. J. Reprod. Fert. Supple. 27, 457-463.

Hull, M. G. R., und Chard, T. (1976): Hormonal aspects of feto-placental function. In: Fetal Physiology and Medicine. Eds R. W. Beard and P. W. Nathanielsz. W. B. Saunders Co, London. pp 371-39.4.

Liggins, G. C., und Kitterman, J. A. (1981): Development of the fetal lung. In: The Fetus and Independent Life. CIBA Foundation Symposium No 86. Ed J. Whelan, Pitman, London. pp 308-330.

Mitchell, M. D., Ellwood, D. A., Robinson, J. S., Anderson, A. B. M., und Turmbull, A. C. (1979): Prostaglandins and parturition in sheep I. N. S. E. R. M. Symposium on prostaglandins and reproductive physiology. Montpellier, Sept. 1979, Abs 13.

Moog, F. (1970): Enzyme development and functional differentiation in the fetus. In: Foetal Growth and Development. Eds H. A. Waisman and G. R. Kerr. McGraw Hill, New York. pp 29-48.

Moss, G. E., Estergreen, V. L., Becker, S. R., und Grant, B. D. (1979): The source of the $5 \alpha$-pregnans that occur during gestation in mares. J. Reprod. Fert. Supple. 27, 511-519.

Nathanielsz, P. W., Rossdale, P. D., Silver, M., und Comline, R. S. (1975): Studies on fetal neonatal and maternal cortisol metabolism in the mare. J. Reprod. Fert. Supple. 23, 625-630.

Nett, T. M., Holtan, D. W., und Estergreen, V. L. (1975): Levels of LH, prolactin and oestrogens in the serum of post partum mares. J. Reprod. Fert. Supple. 23, 201-206.

Nuwahid, B., Brinkham, C. R., Woods, J. R., Martinek, H., und Assali, N. S. (1975): Effects of oestrogens on systemic and regional circulations in normal and renal hypertensive sheep. Am. J. Obstet. Gynec. 123, 495-501.

Pashen, R. L. (1981): Studies on the endocrinology of pregnancy in the mare. PhD thesis, University of Cambridge.

Pashen, R. L., und Allen, W. R. (1979): The role of the fetal gonads and placenta in steroid production, maintenance of pregnancy, and parturition in the mare. J. Reprod. Fert. Supple. 27, 499-509.

Pashen, R. L., Sheldrick, E. L., Allen, W. R., und Flint, A. P. F. (1982): Dehydroepiandrosterone synthesis by the fetal foal and its importance as an oestrogen precursor. J. Reprod. Fert. Supple. 32, 389-397.

Pupkin, M. J., Schomberg, D. W., Nagey, D. A., und Crenshaw, C. (1975): Ef fects of exogenous dehydroepiandrosterone upon the fetoplacental biosynthesis of oestrogens and its effect upon uterine blood flow in the term pregnant ewe. Am. J. Obstet. Gynec. 121, 227-232.
Purvis, A. D. (1978): The induction of labor in mares as a routine breeding farm procedure. Proc. 23rd Ann. Conv. Am. Ass. equine Pract. pp. 145-158.

Raeside, J. I., Liptrap, R. M., McDonell, W. N., und Milne, F. J. (1979): A precursor role for DHA in a feto-placental unit for oestrogen formation in the mare. J. Reprod. Fert. Supple. 27, 493-497.

Rance, T. A., und Park, B. K. (1978): The measurement of oestrone, equilin and dehydroepiandrosterone in the peripheral plasma of pregnant pony mares by radioimmunoassay. J. Steroid Biochem. 9, 1065-1069.

Resnik, R., Killam, A. P., Battaglia, F. C., Makowski, E. E., und Meschia, G. (1974): The stimulation of uterine blood flow by various oestrogens. Endocrin. 94, 1192-1196.

Rosenfeld, C. R., und Worley, R. J. (1978): The effect of systemic infusions of dehydroepiandrosterone on the distribution of uterine blood flow in ovine pregnancy. Am. J. Obstet. Gynec. 130, 385-390.

Rossdale, P. D., Pashen, R. L., und Jeffcott, L. B. (1979): Studies on the use of the synthetic prostaglandin analogue (fluprostenol) to induce foaling. J. Reprod. Fert. Supple. 27, 521-529.

Rossdale, P. D., und Ricketts, S. W. (1974): The Practice of Equine Stud Farm Medicine, Bailliere-Tindall, London.

Rossdale, P. D., Silver, M., Ellis, L., und Frauenfelder, H. (1982): Response of the adrenal cortex to tetracosactrin $\left(\mathrm{ACTH}_{1-24}\right)$ in the premature and full term foal. J. Reprod. Fert. Supple. 32, 545-553.

Seamans, K. W., Harms, P. G., Atkins, D. T., und Fleeger, J. L. (1979): Serum levels of progesterone, $5 \alpha$-Dihydro-progesterone and Hydroxy- $5 \alpha$ pregnanones in the pre-partum and post-partum equine. Steroids 33 , $55-63$.

Sharma, O. P. (1975): Prostaglandin F-2 $\alpha$ in foaling mares. J. Reprod. Fert. 45, 541-543.

Silver, M. (1980): Animal Models in Fetal Medicine. Ed P. W. Nathanielsz. Eds U. Vier, North Holland. pp. 107-132.

Silver, M., Barnes, R. J., Comline, R. S., Fowden, A. L., Clover, L., und Mitchell, M. D. (1979): Prostaglandins in maternal and fetal plasma and in allantoic fluid during the second half of gestation in the mare. J. Reprod. Fert. Supple. 27, 531-539.

Squires, E. L., und Ginther, O. J. (1975): Follicular and luteal development in pregnant mares. J. Reprod. Fert. Supple. 23, 429-433.

Starka, L., und Breuer, H. (1966): Biogenese von $\mathrm{C}_{18}$-Steroiden beim Pferd. Hoffe Seyler Z Physiol. Chem. 344, 124-139.

Stewart, D. R., und Stabenfeldt, G. H. (1981): Relaxin activity in the pregnant mare. Biol. Reprod. 25, 281-289.

Stewart, D. R., Stabenfeldt, G. H., und Hughes, J. P. (1982): Relaxin activity in foaling mares. J. Reprod. Fert. Supple. 32, 603-609.

Tait, A. D., Santikarn, S., und Allen, W. R. (1983): Identification of $3 \beta$-hydroxy-5,7-pregnandien-20-one and $3 \beta$-hydroxy-5,7-androstadien-17-one as endogenous steroids in the fetal horse gonad. J. Endocrin. 99, 87-92.

Thorburn, G. D., und Challis, J. R. G. (1979): Endocrine control of parturition. Physiol. Rev. 59, 863-918.

Thorburn, G. D., Nicol, D. H., Bassett, J. M., Shutt, D. A., und Cox, R. I. (1972): Parturition in the goat and sheep; changes in corticosteroids, progesterone, oestrogens and prostaglandin F. J. Reprod. Fert. Supple. $16,61-84$.

Urwin, $V$. E., und Allen, $W . R$. (1982): Pituitary and chorionic gonadotrophic control of ovarian function during early pregnancy in equids. J. Reprod. Fert. Supple. 32, 371-381.

\section{R. L. Pashen, DVM}

Department of Reproduction

School of Veterinary Medicine

University of California

Davis, California 95616

Erschienen in EQUINE VETERINARY JOURNAL (1984) 16, 233-238. Übersetzt und veröffentlicht mit freundlicher Genehmigung der British Equine Veterinary Association. 